\title{
COMUNIDADES VIRTUALES, GRUPOS Y PROYECTOS DE INVESTIGACIÓN SOBRE IMS LEARNING DESIGN. STATUS QUO, FACTORES CLAVE Y RETOS INMEDIATOS
}

\section{(Virtual communities, research groups and projects on IMS Learning Design. State of the art, key factors and forthcoming challenges)}

Article record

$\underline{\text { About authors }}$

HTML format

\author{
Daniel Burgos (Daniel.Burgos@ou.nl) \\ Rob Koper (rob.koper@ou.nl)
}

\section{Ficha del artículo \\ $\underline{\text { Sobre los autores }}$ \\ Formato HTML}

\begin{abstract}
We carry out a report showing the state of the art about virtual communities, research groups and projects focused on the e-learning specification IMS Learning Design or directly related to it. This specification is currently becoming the most flexible and supported de facto standard for modelling full learning processes, as a complement for any structure of educational contents. Afterwards, as a consequence of the previous study, we develop a reading and a further analysis of the current panorama, and describe the key factors that show the relevance and impact of IMS Learning Design and also the main forthcoming challenges.
\end{abstract}

\section{Keywords}

IMS Learning Design, e-learning standard, interoperability, re-use, unit of learning

\section{Resumen}

Realizamos una revisión sobre el estado de la cuestión en el tema de las comunidades virtuales, los grupos de trabajo y los proyectos de investigación centrados en la especificación de e-learning IMS Learning Design o desarrollados en torno a ella. Esta especificación se está convirtiendo de facto en el estándar más versátil y respaldado para modelar procesos completos de aprendizaje como complemento de estructuras de contenidos educativos. Posteriormente, y como consecuencia del estudio, desarrollamos un análisis y lectura del panorama actual con una indicación de los factores clave que muestran su impacto y relevancia y los principales retos a abordar en un futuro inmediato

\section{Descriptores}

IMS Learning Design, estándar e-learning, interoperabilidad, reutilización, unidad de aprendizaje

\section{INTRODUCCIÓN}

A la luz del movimiento que está surgiendo en los últimos años en torno a la formación online $y$, en concreto, a la estandarización de la misma, diversos grupos internacionales vienen desarrollado una interesante actividad de investigación, producción y/o divulgación al respecto. Especificaciones como SCORM (ADL, 2000), IMS content Packaging (IMS, 2001) o IMS 
Burgos, D. y Koper, R. (2005). Comunidades virtuales, grupos y proyectos de investigación sobre IMS Learning Design. Status quo, factores clave y retos inmediatos. RELIEVE: v. 11, n. 2, p. 189-200.

http://www.uv.es/RELIEVE/v11n2/RELIEVEv11n2_6.htm

Simple Sequencing (IMS, 2003) son un buen punto de partida para la estructuración de contenidos de aprendizaje o de otra índole. Entre todas ellas, IMS Learning Design (o simplemente LD) (IMS, 2003a) aparece como el siguiente paso lógico permitiendo no ya sólo el empaquetamiento de recursos sino el modelado completo de escenarios de aprendizaje y la aplicación de diversos enfoques pedagógicos (Koper y Tattersall, 2005 y Burgos et al, 2005). De esta manera, tanto el profesor como el pedagogo pueden migrar cursos presenciales a plataformas online, garantizado la interoperabilidad y la reutilización de los paquetes educativos generados.

Con la intención de proporcionar una ligera visión general sobre el panorama actual en cuanto a comunidades virtuales, grupos de trabajo y proyectos de investigación centrados en la especificación IMS Learning Design o desarrollados en torno a ella, aportamos un completo directorio con una breve descripción y un enlace asociado con información más detallada en el idioma de origen, cuando sea posible. Para finalizar, y como conclusión, analizamos el estado actual de la cuestión, los factores clave que muestran su impacto y relevancia y los retos a abordar en un futuro inmediato.

Este documento está desarrollado dentro del marco del proyecto europeo UNFOLD ${ }^{[1]} \mathrm{y}$ del proyecto Learning Network for Learning Design ${ }^{\text {[2] }}$ de la Open University of The Netherlands ${ }^{[3]}$.

\section{Categorías}

Para detallar el estado de la cuestión hemos dividido los grupos en las siguientes categorías:

1. Grupos que trabajan para depurar, modificar, ampliar y/o mejorar las especificaciones en sí mismas.

2. Grupos que utilizan las especificaciones existentes para adaptarlas a su realidad concreta desde un enfoque práctico específico.

3. Proyectos y comunidades virtuales de aprendizaje no estructurado que intentan acercar las especificaciones a un público objetivo concreto y buscan divulgarla para su mayor y mejor diseminación.

4. Grupos y proyectos que trabajan con diversas especificaciones sobre e-learning, utilizándolas como inspiración y soporte, que no se someten a ninguna de ellas y aportan su propio sistema de modelado.

5. Grupos que se centran en desarrollar aplicaciones informáticas que interpreten las especificaciones y que permitan una utilización sencilla y potente para el mayor número posible de personas.

6. Grupos multidisciplinares que realizan varias o todas las tareas anteriores.

\section{Descripción de Comunidades virtuales, grupos y proyectos de investigación}

Pasamos a desarrollar brevemente cada una de las categorías:

1) Grupos que trabajan para depurar, modificar, ampliar y/o mejorar las especificaciones en sí mismas

\section{a. CETIS (http://www.cetis.ac.uk/)}

Representa la educación secundaria y universitaria en los organismos de estándares educativos, tales como IMS, CEN/ISSS o IEEE LTSC. Se encarga de asesorar a estas instituciones sobre las implicaciones estratégicas, técnicas y pedagógicas de la adopción de estándares en tecnología educativa. También asesora técnicamente a los diversos projectos y programas de JISC, the Joint Information Systems Committee of the Higher and Further Education Funding Councils (ver referencia más adelante en esta sección) y a los programas sobre e-learning de la Unión Europea.

CETIS trabaja distribuido en diversos grupos en torno a diversas especificaciones, incluyendo metadata, contenido educativo, cuestionarios, accesibilidad y otros .

CETIS está financiado por JISC y gestionado por la Universidad de Bolton (ver más adelante)

\section{b. JISC (http://www.jisc.ac.uk/)}

The Joint Information Systems Committee of the Higher and Further Education Funding Councils 
Burgos, D. y Koper, R. (2005). Comunidades virtuales, grupos y proyectos de investigación sobre IMS Learning Design. Status quo, factores clave y retos inmediatos. RELIEVE: v. 11, n. 2, p. 189-200.

http://www.uv.es/RELIEVE/v11n2/RELIEVEv11n2_6.htm

(JISC) da apoyo a los sistemas educativos de secundaria y universitarios asesorando sobre las oportunidades y consecuencias de utilizar tecnologías de comunicación e información en la enseñanza, el aprendizaje, la investigación y la gestión administrativa. JISC trabaja mediante un comité de expertos (directores, gestores, académicos y tecnólogos) de educación secundaria y superior y proporciona nuevos entornos educativos, acceso a recursos electrónicos, redes de aprendizaje, guías para cambio institucional, asesoría y soporte regional a colegios

\section{c. The Open Group}

\section{(http://www.opengroup.org/)}

Consorcio comercial y tecnológicamente neutral que proporciona acceso a información coordinada entre diferentes proyectos y aplicaciones basados en estándards abiertos y en interoperabilidad. Trabaja con clientes, proveedores y consorcios para detectar, comprender y solucionar necesidades del sector. También facilita la interoperabilidad entre las partes implicadas, la integración de diferentes especificaciones y los desarrollos de código abierto. Básicamente, es un foro de intercambio de ideas entre miembros de alto nivel, mezclando el mundo académico y el empresarial, centrados en tecnología educativa y en e-learning standards

\section{d. Telcert (www.opengroup.org/telcert)}

Projecto de desarrollo e investigación amparado en el Sexto Programa Marco de la Unión Europea, está formado por un consorcio de proveedores de contenidos, investigación y organizaciones industriales y desarrolla aplicaciones informáticas para comprobar la interoperabilidad de de los sistemas e-learning, tanto en contenido como en tecnología. Una parte importante de su trabajo es generar perfiles de interoperabilidad y reusabilidad que permitan ser adoptados por las aplicaciones informáticas y las herramientas de re-ingeniería

2) Grupos que utilizan las especificaciones existentes para adaptarlas a su realidad concreta desde un enfoque práctico específico

a. Laboratory for Ontological Research (LORE) (http://lore.iat.sfu.ca/)

Perteneciente a la Simon Fraser University (Canada) y dirigido por Griff Richards, este grupo de investigación centra sus esfuerzos en la gestión de conocimiento y más concretamente en ontologías, interoperabilidad y razonamiento. Modelan sistemas de aprendizaje online, construyen sistemas distribuidos e interoperables, entornos adaptativos y compartición de conocimiento. Su última investigación (el ECL Interoperability Center) desarrolla una infraestructura para repositorio de objetos de aprendizaje, coleccionando información técnica, componentes y servicios sobre interoperabilidad.

b. Universidad de Vigo (http://wwwgist.det.uvigo.es/ maeiro/thesis.html)

El Grupo de Ingeniería de Sistemas Telemáticos de la Universidad de Vigo (España) desarrolla una investigación sobre re-utilización e interoperabilidad de unidades de aprendizaje escritas en IMS Learning Design y generalizadas en cualquier lenguaje de modelado educativo o EML. Particularmente, proponen extensiones a la especificación intentando solventar algunas deficiencias que encuentran ante situaciones concretas de enseñanza. Paralelamente desarrollan una herramienta que valore la capacidad de modelado pedagógico de un EML y un editor que utilice las extensiones propuestas para demostrar la validez de las mismas y su aplicación real a los escenarios pedagógicos que no son completamente realizables con la especificación actual.

c. Laboratorio DEI, proyecto CASLO (http://caslo.dei.inf.uc3m.es/)

El DEI, perteneciente a la Universidad Carlos III (España), desarrolla el proyecto CASLO (Collaborative Annotation Service for Learning) dirigido por Juan Manuel Dodero. CASLO permite compartir la autoría sobre recursos de aprendizaje, habilitando secciones de código XML anotadas, que pueden ser comentadas y modificadas por el grupo de trabajo desde distintos puntos de acceso (al estilo de un sistema wiki) dentro de un tiempo determinado para, finalmente, proceder a la incorporación y validación consensuada del contenido final. El sistema puede trabajar con cualquier recurso basado en XML, como objetos LOM o IMS Learning Design.

\section{d. Universidad de Valladolid}


Burgos, D. y Koper, R. (2005). Comunidades virtuales, grupos y proyectos de investigación sobre IMS Learning Design. Status quo, factores clave y retos inmediatos. RELIEVE: v. 11, n. 2, p. 189-200.

http://www.uv.es/RELIEVE/v11n2/RELIEVEv11n2_6.htm

\section{(http://gsic.tel.uva.es/index.php?lang=es)}

Este grupo de investigación, con sede en la Escuela Técnica Superior de Ingenieros de Telecomunicación de Valladolid, tiene como objetivo trabajar en los campos de los Sistemas Inteligentes, que incluyen redes neuronales y sistemas neuro-difusos, con especial interés en los modelos derivados de la Teoría de Resonancia Adaptativa (ART); los Sistemas Cooperativos, es decir en CSCW (Computer Supported Cooperative Work) con especial énfasis en CSCL (Computer Supported Collaborative Learning) y el proyecto TELL, financiado por la Unión Europea dentro de su programa e-learning.

El proyecto TELL se enfoca en la aproximación pedagógica y didáctica del aprendizaje colaborativo apoyado por ordenador/red (CSCL o NSCL). Es un esfuerzo metódico y sistemático para apoyar la comprensión de los procesos de aprendizaje que suceden en entornos CSCL mediante patrones de diseño, realizar un meta-estudio de métodos y herramientas que miden la efectividad de procesos CSCL, ofrecer y proponer métodos y herramientas - toolkits para educadores que quieren medir la eficiencia de actividades CSCL, ofrecer medios para la formación de los actores humanos involucrados (o de los que quieren involucrarse) en actividades de aprendizaje colaborativo y apoyar el diseño de nuevas herramientas tecnológicas efectivas para aprendizaje colaborativo.

Uno de los proyectos más representativos en torno a IMS LD es Collage (http://gsic.tel.uva.es/collage), una herramienta que de autor para aprendizaje colaborativo que trata de ayudar al diseñador de escenarios didácticos proporcionando una serie de patrones que puedan ser utilizados como base.

\section{e. Grup de Tecnologies Interactives} (www.tecn.upf.es/gti)

Perteneciente a la Universidad Pompeu Fabra (España) y dirigido por Josep Blat este grupo centra su investigación en diferentes aspectos sobre interactividad multimedia e Internet. Entre otras áreas se encuentra el desarrollo de software y servicios web, la consultoría de usabilidad, las aplicaciones de dispositivos móviles, los juegos y el e- learning. Trabaja en los proyectos UNFOLD y SCOPE (ver referencia más adelante) además de una docena más de iniciativas financiadas por la Unión Europea.

\section{f. LT3 Centre (http://lt3.uwaterloo.ca/)}

Perteneciente a la University of Waterloo (Canadá) y dirigido por Liwana Bringelson, desarrolla y comparte diseños instructivos innovadores, diseña y apoya comunidades virtuales de aprendizaje no estructurado, crea objetos de aprendizaje y colabora en repositorios, mide y analiza impactos de la tecnología en la enseñanza y en el aprendizaje. Concretamente, desarrolla el modelo T5 (tareas, tutorización, trabajo en equipo, recursos y herramientas) de aprendizaje y enseñanza, que predica el aprendizaje colaborativo entre los diversos miembros implicados en un procedo educativo. Esta teoría respalda la aplicación LearningMapR que, a su vez, trabaja sobre el concepto de plantillas de aprendizaje y unidades de aprendizaje (UoL), es decir, elementos reusables que definen casos de estudio y metodologías pedagógicas y que se ofrecen a la comunidad para su uso y adaptación contextual concretas.

\section{g. Salerno University (http://www.unisa.it/)}

Participa en el proyecto ELeGI de la UE, construyendo unidades de aprendizaje (UoLs) personalizadas para un determinado estudiante, según su estado cognitivo preciso y las preferencias personalizadas, la pedagogía utilizada, la retroalimentación del usuario. Reuniendo toda esta información y administrándola mediante agentes informáticos, el proceso de personalización en el proceso de aprendizaje es máximo. Actualmente se trabaja en la posibilidad de implementar toda la teoría utilizando diversos estándares, IMS Learning Design entre ellos, potenciando un documento base o manifiesto que refleje adecuadamente la metodología.

3) Proyectos y comunidades virtuales de aprendizaje no estructurado que intentan acercar las especificaciones a un público objetivo concreto y buscan divulgarla para su mayor y mejor diseminación

a. UNFOLD (http://www.unfold-project.net/, Europa) 
Burgos, D. y Koper, R. (2005). Comunidades virtuales, grupos y proyectos de investigación sobre IMS Learning Design. Status quo, factores clave y retos inmediatos. RELIEVE: v. 11, n. 2, p. 189-200.

http://www.uv.es/RELIEVE/v11n2/RELIEVEv11n2_6.htm

Understanding New Frameworks of Learning Design (UNFOLD) es un proyecto del Sexto Programa Marco de la UE y se centra en la implementación y uso de estándares sobre e-learning, tanto para usuarios individuales como para actividades multi-usuario, y en aplicaciones de código abierto. Otros temas de interés son la interoperabilidad de los productos, contenidos y plataformas de aprendizaje online y los modelos pedagógicos soportados por ellas. Su dinámica de trabajo se centra en las comunidades virtuales o de práctica en torno a diversos temas específicos para profesores, escritores, pedagogos, investigadores y programadores.

El proyecto UNFOLD es clave para entender el planteamiento y desarrollo de esta tesis ya que constituye el centro de operaciones, experimentos y trabajo de campo sobre comunidades virtuales y participación de usuarios.

b.

R2R

(http://commons.ucalgary.ca/weblogs/learningdesi gn, Canadá)

Repository to Reality (R2R) se fundamenta en el trabajo con comunidades virtuales en torno a la especificación de e-learning IMS Learning Design (IMS LD). Sub-grupos de interés en recomendaciones, herramientas y uso trabajan internamente y de manera descentralizada para proporcionar una base de conocimiento a investigadores y desarrolladores de la especificación, con el objeto de aplicarla y configurarla contextualmente.

Diversas universidades canadienses investigan sobre implicaciones técnicas y pedagógicas en la implementación de IMS LD, desarrollan un vocabulario limitado y escriben una serie de informes sobre los retos de implementar la especificación según el contexto.

\section{c. ACETS (http://www.acets.ac.uk/, Reino Uni-} do)

Este proyecto colaborativo desarrolla y evalua procesos para implementar el uso de objetos de aprendizaje reutilizables en el mundo médico, fundalmentalmente en el gremio de los doctores. Aunque los alumnos de Medicina son el principal objetivo, también se pretende que los profesores utilicen estos objetos de aprendizaje para retroalimentarse y aplicarlos en sus clases. Se centra, por tanto, en lograr procesos más sencillos y rápidos, así como más satisfactorios intelectual y educativamente hablando, en la utilización de objetos de aprendizaje. ACETS es un proyecto financiado por JISC y utiliza la generación de taxonomías educativas y su aplicación en una variedad de escenarios para lograr procedimientos estándar que sean fácilmente exportables y utilizables por otros objetos de aprendizaje o escenarios.

\section{d. SCOPE}

(http://www.tecn.upf.es/scope/showcase, Europa)

Aunque el proyecto SCOPE finalizó el 30 de Junio de 2003, sus enlaces continúan activos y son fuente de consulta y comunicación. Mantiene una red de comunidades virtuales en diversos países de Europa con el objetivo de reutilizar derechos de propiedad intelectual científicos y convertirlos en objetos de aprendizaje y productos de alto valor. Para ello, se hacen disponibles contenidos mediante un servicio de suscripción web, se establecen mecanismos de búsqueda y recuperación de información multilingüe y se reutilizan contenidos particularizados para los profesionales médicos.

\section{e. Pool (http://www.edusplash.net/, Canadá)}

El Portal for Online Objects in Learning es un consorcio de diversas organizaciones, públicas y privadas, del sector educativo que desarrollan repositorios o bases de datos de objetos de aprendizaje. Para ello se trabaja tanto con la definición de arquitecturas locales basadas en metadatos (en concreto, el estándar IEEE LOM), como la realización de productos software y hardware que permitan alimentar dichas estructuras con contenido real. El producto que concreta todos los esfuerzos de esta comunidad virtual de trabajo colaborativo en torno a los objetos de aprendizaje y la categorización es la aplicación Splash, que en su última versión permite un vínculo con Edusorce (ver abajo). Finalizado en 2002

\section{f. Edusource (http://www.edusource.ca/, Cana-} dá)

Proyecto colaborativo que pretendía (finalizó en 2004) generar una red repositorios interconectados e interoperables sobre objetos de aprendizaje a lo largo de Canadá. Su objetivo también era liderar el desarrollo de software, sistemas, protocolos y 
Burgos, D. y Koper, R. (2005). Comunidades virtuales, grupos y proyectos de investigación sobre IMS Learning Design. Status quo, factores clave y retos inmediatos. RELIEVE: v. 11, n. 2, p. 189-200.

http://www.uv.es/RELIEVE/v11n2/RELIEVEv11n2_6.htm

prácticas que permitieran explotar y mantener dicha red. Como proyecto bilingüe (inglés y francés) que permite la comunicación y el trabajo colaborativo entre todas las provincias francesas, precisaba de una conexión de banda ancha a Internet con capacidad suficiente para envíos masivos de información.

\section{g. Iclass (http://www.iclass.info/, Europa)}

"Intelligent cognitive-based open learning system for schools" es un proyecto que pretende desarrollar un sistema y un entornos abiertos de aprendizaje basado en inteligencia cognitiva, con el objeto de satisfacer las necesidades de alumnos individuales. El producto a obtener será una aplicación basada en una arquitectura ontológica de almacenamiento y recuperación de información que permita generar dinámicamente objetos individuales de aprendizaje. Para ello trabaja con una estructura de comunidad virtual orientada por objetivos y por entornos comunes de trabajo colaborativo.

h. Ladie (www.elframework.org/refmodels/ladie, Reino Unido)

Este proyecto fundado por JISC (ver referencia en esta misma sección) desarrolla un modelo de referencia que fundamenta el diseño y construcción de actividades de aprendizaje, así como el descubrimiento, la especificación, la secuenciación y el empaquetamiento de contenido. Del mismo modo, también sustenta los entornos donde las actividades formativas se llevan a cabo y la ejecución de las actividades formativas en sí mismas. El proyecto LADIE está relacionado íntimamente con DialogPlus (ver referencia más adelante en esta misma sección) con el objetivo de describir y reconocer las necesidades tecnológicas asociadas a este modelo pedagógico de referencia.

\section{i. Lornet (http://www.lornet.org/, Canadá)}

Centrado en la reusabilidad de objetos de aprendizaje diseminados a través de Internet, Lornet trata de definir los mecanismos de interoperabilidad que permitan localizar los elementos buscados y permitir adaptarlos al contexto preciso del usuario final. Para ello se pretende construir uno o varios prototipos centrados en la agregación de repositorios de objetos de aprendizaje multi-idioma y multimedia relacionados entre sí mediante metadatos y un sistema ontológico de almacenamiento y recuperación de recuperación. Lornet utiliza las premisas de ciertas especificaciones centradas en e-learning (fundamentalmente de IMS Global Consortium) para realizar un análisis crítico de las mismas y complementarlas.

j. DialogPlus (http://www.dialogplus.org/, Reino Unido y EEUU)

"Digital Libraries in Support of Innovative Approaches to Learning and Teaching in Geography" es un proyecto financiado por JISC (ver referencia en esta misma sección) que proporciona una estructura de información distribuida para que los graduados y post-graduados de titulaciones sobre Geografía puedan compartir recursos y enriquecerse mutuamente a través del aprendizaje colaborativo en línea y diversas aplicaciones de código abierto centradas en la generación de bibliotecas de recursos ad hoc.

4) Grupos y proyectos que trabajan con diversas especificaciones sobre e-learning, utilizándolas como inspiración y soporte, que no se someten a ninguna de ellas y aportan su propio sistema de modelado

\section{a. Projecto Moodle (http://moodle.org/, Austra-} lia)

Modular Object-Oriented Dynamic Learning Environment, sistema de gestión de cursos (CMS) de código abierto, desarrollado en lenguaje PHP, es un proyecto originalmente particular fundado por Martin Dougiamas que se fundamenta en el constructivismo social (el individuo aprende a medida que interactúa con el entorno y con otros) de una comunidad educativa virtual muy extensa alrededor del mundo. Se desarrolla bajo gestión de licencia pública GNU (Free Software Foundation, 1989), lo que permite a cualquier interesado modificar y adaptar el código de la aplicación para a sus propios intereses siempre y cuando extienda dicha licencia a su creación.

Moodle está traducido a más de cincuenta idiomas y su comunidad virtual es una de las más activas dentro de los educadores y diseñadores de aprendizaje. Actualmente prepara la interoperabilidad entre el sistema LAMS (ver referencia en 
Burgos, D. y Koper, R. (2005). Comunidades virtuales, grupos y proyectos de investigación sobre IMS Learning Design. Status quo, factores clave y retos inmediatos. RELIEVE: v. 11, n. 2, p. 189-200.

http://www.uv.es/RELIEVE/v11n2/RELIEVEv11n2_6.htm

esta misma página) y la especificación de elearning IMS Learning Design.

b. Macquarie University and LAMS Foundation (http://www.lamsinternational.com/, Australia)

Grupo de trabajo que desarrolla LAMS, aplicación académica dirigida por James Dalziel y centrada en el enfoque pedagógico de la construcción de materiales educativos, más que en la creación técnica. Los profesores suelen apreciar la sencillez para secuenciar lecciones y cursor y para diseñar estructuras de aprendizaje. Inspirada en la especificación IMS Learning Design, la próxima versión de LAMS será de código abierto y trabajará sobre la interoperabilidad de sus objetos de aprendizaje con otros sistemas y motores de ejecución.

\section{c. University Pierre et Marie-Curie Paris 6} (Francia)

Monique Baron dirige un grupo de investigación que desarrolla un editor y un simulador basado en las especificaciones Educational Modelling Language (OUNL, 2000) e IMS Learning Design. El objetivo principal es dotar a la comunidad educativa de una herramienta capaz de modelar unidades de enseñanza y utilizar objetos de aprendizaje de una manera sencilla y adaptada a la didáctica propia de cada profesor.

d. LICEf, University of Quebec (www.licef.teluq.uquebec.ca/francais/real/mot.htm , Canadá)

Este grupo de investigación, dirigido por Gilbert Paquette, desarrolla desde antes de la aparición de los estándares en e-learning una metodología de creación de materiales pedagógicos formalizada en la aplicación MOTPlus, y que tiene grandes similitudes con IMS Learning Design, aunque no es completamente compatible. Se basa en la representación gráfica de cualquier metodología didáctica empleada para generar unidades de aprendizaje. Al igual que ocurre con ASK LDT las posibilidades de definición gráfica no permiten la importanción de recursos desarrollados con otras aplicaciones, aunque sí la exportación con un alto porcentaje de satisfacción.

e. Labset, University of Liège (http://www.labset.net/, Bélgica)
Ha desarrollado e implanta la metodología 8LEM, dirigida por Dominique Verpoorten, que se basa en la generación de más de ochenta escenarios pedagógicos típicos que se adaptan y personalizan según las necesidades concretas de cada cliente. Completamente centrado en facilitar al profesorado la adaptación de metodologías de enseñanza al medio online, parte de la creación de plantillas que identifican procesos de aprendizaje reales que son utilizados de manera inmediata por el profesor, sin necesidad de ningún conocimiento tecnológico previo.

\section{f. .LRN (http://www.dotlrn.org/, Internacional)}

Desarrollo de código abierto para aprendizaje e investigación, ampliamente extendido (más de medio millón de usuarios y traducciones a ochenta idiomas) y fácilmente instalable y configurable. Originalmente desarrollado por el MIT (Boston, EEUU) aunque no soporta especificaciones de elearning se basa en los mismos conceptos para generación de estructuras de aprendizaje. Oficialmente permite la aplicación de cualquier tipo de pedagogía y, al igual que Moodle (ver referencia más arriba) fomenta la adaptación y la relación comunitaria virtual para su implementación y soporte.

g. Elive LD Suite (http://www.elive-ld.com/, Alemania)

Desarrollada por cogito GmbH esta aplicación permite la edición, documentación y optimización de escenarios de aprendizaje de manera gráfica. Está basada en la especificación IMS Learning Design y permitirá exportar Unidades de Aprendizaje Level A. El objetivo final de eLive es proporcionar una herramienta visual que permita generar mapas de conceptos, estructuras y métodos, acorde con el modelo pedagógico seleccionado por el diseñador de aprendizaje o profesor.

5) Grupos que se centran en desarrollar aplicaciones informáticas que interpreten las especificaciones y que permitan una utilización sencilla y potente para el mayor número posible de sectores de interés

a. Reload Project (http://www.reload.ac.uk/, Reino Unido) 
Burgos, D. y Koper, R. (2005). Comunidades virtuales, grupos y proyectos de investigación sobre IMS Learning Design. Status quo, factores clave y retos inmediatos. RELIEVE: v. 11, n. 2, p. 189-200.

http://www.uv.es/RELIEVE/v11n2/RELIEVEv11n2_6.htm

Gestionado por la Universidad de Bolton, dirigido por Oleg Liber y financiado por JISC (ver referencia en esta misma sección) Reload desarrolla editores de código abierto, visualizadores y entornos virtuales basados en estándares (IMS Learning Design, IMS Content Packaging y SCORM) con el objetivo de crear y ejecutar unidades y objetos de aprendizaje. Actualmente el editor de IMS CP está completamente activo, así como el editor de SCORM. En referencia a IMS LD, el visualizador no es un player completo, sino que basado en el motor Coppercore (Vogten y Martens, 2004) desarrollado por la Open Univesity of The Netherlands, ejecuta de manera sencilla y mediante usuarios paja las unidades de aprendizaje diseñadas previamente. El editor de IMS LD, por su parte, permite trabajar con los tres niveles de la especificación, aunque el nivel $\mathrm{C}$ no ha sido probado todavía.

b. University of Duisburg (http://www.unfoldproject.net/general_resources_folder/cosmos_tool.zip, Alemania)

Desarrolla un producto llamado Duisburg Collaborative Learning Authoring System que permite el diseño de unidades de aprendizaje mediante un sistema de notación propio XML, aportando elementos condicionales, propiedades y disparadores asociados a eventos concretos. La gran similitud con IMS Learning Design y la necesidad de asociarse con una especificación estándar ya operativa ha llevado a sus creadores, Young Wu Miao and Kai Hoeksema, a adaptarlo rápidamente hasta nivel B, creando COSMOS

c. Advanced e-Services for the Knowledge Society

(www.certh.gr/\&en/home/index.htm, Grecia)

Dentro del Informatics and Telematics Institute (University of Piraeus), Demetrios Sampson dirige el ASK, unidad de investigación que se centra en la creación de software educativo basado en estándares y metadatos. Desarrolla un producto denominado ASK LDT (http://www.ask.iti.gr/) que permite la creación de unidades de aprendizaje bajo la especificación IMS Learning Design y el ASK LOM-RM que gestiona repositorios de objetos de aprendizaje.

\section{d. Cepiah (www.hds.utc.fr/cepiah, Francia)}

Grupo de investigación ubicado en la Université de Technologie de Compiègne - Centre de Recherches de Royallieu y centrado en el desarrollo de módulos que permiten la mejora y desarrollo del diseño, evaluación y modelos de enseñanza dentro de los sitios web centrados en formación. Con el objeto de ayudar a los profesores sin bagaje técnico, se ha creado netUniversité, compuesto por un editor, un generador de sitios web y un módulo de administración. De esta manera, se pretende abarcar todo el ciclo de generación de escenarios de aprendizaje online, desde la concepción hasta la ejecución real en clase.

6) Grupos multidisciplinares que realizan varias o todas las tareas anteriores

a. Educational Technology Expertice Center, OTEC （www.ou.nl/eCache/DEF/5/071.html, Holanda)

Dentro de la Open University of The Netherlands este instituto de investigación se centra en la tecnología educativa (evaluación, cursos, retroalimentación, navegación). Más concretamente el Departamento de Desarrollo, dirigido por Rob Koper, desarrolla proyectos centrados en estándares. Fue el creador del Educational Modelling Language que sirvió como base para la implementación de la especificación IMS Learning Design, base de gran parte de la discusión actual sobre estándares en e-learning. Actualmente, además de proyectos de investigación sobre navegación (ROMA, http://www.ou.nl/eCache/DEF/13/374.html), comunidades virtuales (LN4LD, http://moodle.learningnetworks.org/), aprendizaje adaptativo y retroalimentación (PET) y un largo etcétera, trabaja en la generación de motores de ejecución para especificaciones (Coppercore, http://www.coppercore.org/), editores (Copperauthor, http://www.copperauthor.org/), entornos virtuales de aprendizaje (Alfanet, http://alfanet.ia.uned.es/ y Edubox), navegadores (Sled, http://ldplayer.sourceforge.net/), además de colaborar con casi todos los grupos de investigación anteriormente mencionados en esta página. Paralelamente, mantiene grupos de depuración y ampliación de especificaciones IMS (Content Packaging, Learning Design, QTI...), interopera- 
Burgos, D. y Koper, R. (2005). Comunidades virtuales, grupos y proyectos de investigación sobre IMS Learning Design. Status quo, factores clave y retos inmediatos. RELIEVE: v. 11, n. 2, p. 189-200.

http://www.uv.es/RELIEVE/v11n2/RELIEVEv11n2_6.htm

bilidad (con SCORM, Moodle y LAMS), desarrolla una amplia labor divulgativa en proyectos (UNFOLD, Prolearn, Scope), conferencias (Online Educa Berlin, Online Educa Madrid, SIGOSSE, ICALT...), publicaciones (JCAL, ILE, JIME, ET\&S...), cursos y talleres (Campus Virtual, Eucen...), consorcios oficiales de regulación (IMS, IEEE...) y un largo etcétera. Actualmente, constituye uno de los principales centros de investigación, desarrollo, debate y divulgación sobre tecnología educativa.

b. Institute of Educational Technology, IET (http://iet.open.ac.uk/, Reino Unido)

Dentro de la Open University of The United Kingdom, asesora sobre la utilización de las tecnologías modernas en la optimización del aprendizaje a distancia y online. Colabora con más de cincuenta proyectos nacionales e internacionales sobre tecnología educativa, investigación sobre aprendizaje e e-learning. Gracias a su inclusión dentro de la Open University lleva a cabo experimentos y desarrollos con alumnos/as que permiten un análisis de primera mano sobre casos reales, lo que revierte una mejora y depuración de los métodos y sistemas utilizados. Participa activamente en proyectos y comunidades virtuales sobre estándares de e-learning (UNFOLD) y en la generación de aplicaciones de aprendizaje (UoL) basadas en estándares, como es el caso del visualizador SLED de unidades de aprendizaje desarrolladas en IMS Learning Design, desarrollado al amparo del motor Coppercore, y soportando UoLs de nivel A. También es el caso del proyecto ELF Toolking (www.elearning.ac.uk/news_folder/phase2toolkits ), en el que trabajan Alex Little y Patrick McAndrew, que pretende lograr una integración de especificaciones IMS (LD y QTI principalmente) o del proyecto

Demonstrator (www.elearning.ac.uk/news_folder/toolkitdemonst rators), que pretende mostrar usos y características de la especificación IMS Learning Design a través de ejemplos concretos que puedan ser reutilizados.

\section{Análisis y conclusión}

Más de treinta grupos y proyectos de investigación internacionales en torno a IMS Learning Design o directamente relacionados con ella mues- tran un panorama alentador para esta joven especificación. Desde el motor de ejecución Coppercore hasta los visualizadores de Reload o Sled pasando por la media docena de editores desde un punto de vista tecnológico se puede comprobar que IMS Learning Design está respaldada y despierta el interés fundamentalmente del sector académico (Open University of The Netherlands, Open University of the United Kingdom, Universities of Duisburg, Piraeus, Valladolid, Vigo...) pero también del sector comercial (eLive, 8Lem...). No obstante, y además de este interés, hay tres factores clave que muestran su impacto y relevancia. Primero, el número de aplicaciones inspiradas en LD o que soportan la exportación de sus paquetes de información en paquetes LD. Los proyectos actuales centrados exclusivamente en LD (CopperCore, Reload LD Editor y LD Player, netUniversité, CopperAuthor...) se ven reforzados por otras aplicaciones sobre e-learning que comenzaron inspiradas en LD o que nacieron antes y que han considerado la migración o incorporación de LD en su modelo conceptual (Lams, Ask, MotPlus...). Dentro del reciente movimiento sobre estandarización del e-learning parece que el enfoque de LD, a su vez basado en el Educational Modelling Language (EML), es fuente de inspiración y desarrollo, pero también de contraste y confrontación, utilizándose para depurar y mejorar una aproximación a la generación y uso de escenarios de aprendizaje.

Segundo, y quizá más decisivo, el número de comunidades virtuales que surgen en torno a este tema es amplio y continua incrementándose. Generalmente auspiciadas por proyectos financiados por organismos oficiales (UNFOLD, Ladie, Lornet, iClass...) pero también con alguna representación espontánea proveniente de foros de discusión ya activos (Moodle), los temas recurrentes del elearning nutren los foros de estas comunidades, impulsando el debate crítico sobre características, utilidad y funcionalidades de las especificaciones, sus fundamentos teóricos y las aplicaciones informáticas relacionadas. Estas comunidades representan el interés generalizado y recurrente de los grupos habituales de usuarios finales (fundamentalmente profesores, proveedores de contenidos y diseñadores de aprendizaje, pero también desarro- 
Burgos, D. y Koper, R. (2005). Comunidades virtuales, grupos y proyectos de investigación sobre IMS Learning Design. Status quo, factores clave y retos inmediatos. RELIEVE: v. 11, n. 2, p. 189-200.

http://www.uv.es/RELIEVE/v11n2/RELIEVEv11n2_6.htm

lladores de sistemas e investigadores) en mejorar su formación online y adaptar la formación presencial a plataformas telemáticas rentabilizando al máximo el tiempo y el esfuerzo requeridos y logrando unidades fácilmente interoperables y reutilizables.

Tercero y último, la internacionalización. Aunque IMS nació en Estados Unidos, como SCORM, su presencia en Europa es cada vez mayor y el interés demostrado por países como Holanda, España, Reino Unido, Portugal, Francia, Italia, Alemania y un largo etcétera es amplio. Del mismo modo, y fuera de Europa, Canadá, Australia, Rusia, Brasil o Marruecos representan sólo una pequeña muestra de la extensión y diversidad de la especificación.

Si juntamos los tres factores, aplicaciones LD, comunidades virtuales en activo y dispersión geográfica, contamos con un núcleo de actuación fuerte, respaldado por el mundo académico y por los usuarios potenciales con diversos emplazamientos.

Por otra parte, y lejos de ser una concepción estancada, LD y el resto de especificaciones en torno al e-learning, tienen una serie de retos que abordar cuanto antes. Primero, aunque hoy en día únicamente la exportación a otras especificaciones parece posible, incluso para el editor más avanzado (fijándonos por ejemplo en Reload LD Editor) la importación al 100\% representa todavía un obstáculo y constituye por tanto un gran paso para lograr el intercambio efectivo de paquetes de información. De esta manera, se logrará uno de los objetivos de la estandarización del e-learning, como es la interoperabilidad de las unidades o actividades de aprendizaje en diversos sistemas de edición y ejecución, con el objeto de lograr flexibilidad y un mayor grado de autonomía. Del mismo modo, junto con la interoperabilidad, el intercambio de paquetes logrará la re-utilización de unidades de aprendizaje previamente modeladas, bien para su re-definición, bien para su compilación e inclusión en unidades mayores.

Segundo, editores o herramientas de autoría con un diseño gráfico y de información más centrados en el usuario final y menos en los logros técnicos. Es cierto que este último año hemos pasado de no contar con ninguna aplicación basada en LD a tener más de una docena centradas o en torno a $\mathrm{LD}$, lo que supone un gran avance; pero el punto en común de todas ellas es la realización técnica de lo que la especificación define, no la utilización sencilla por usuarios reales. Es decir, con las aplicaciones informáticas actuales se pueden construir unidades de aprendizaje, mayormente sencillas o en nivel A, pero el grado de conocimientos técnicos necesarios es alto y la facilidad de uso de los interfaces es escasa. Es necesario un interfaz con un mayor grado de usabilidad, con una metáfora de aplicación y un sistema de diseño gráfico drag\&drop, además de una ayuda contextual y bien documentada, en vez de un sistema de solapas y etiquetas para rellenar sin mayor soporte ni información y sin una conexión educativamente metodológica entre ellas, que es lo que existe en la actualidad.

Tercero y último, un campus virtual (Learning Management System -LMS- o Virtual Learning Environment -VLE-) desarrollado bajo cánones de LD o compatible con Unidades de Aprendizaje creadas en LD, con posibilidad de intercambio (importación y exportación) y ejecución y una capa de servicios que permita interactuar con ellas. Servicios como foros, chats, seguimiento de expediente, trabajo colaborativo, servicio de noticias o correo, por citar algunos, arroparían el núcleo LD.

\section{Referencias}

IMS (2001) Content Packaging. Boston, USA : IMS Global Consortium. Disponible en: http://www.imsglobal.org/. Último acceso: 5 de agosto de 2005]

IMS (2003) Simple Sequencing. Boston, USA : IMS Global Consortium. Disponible en: http://www.imsglobal.org/. Último acceso: 5 de agosto de 2005

IMS (2003a) IMS Learning Design. Information Model, Best Practice and Implementation Guide, XML Binding, Schemas. Version 1.0 Final Specification. Boston, USA : IMS Global Consortium. Disponible en: www.imsglobal.org/content/learningdesign. Último acceso: 5 de agosto de 2005

ADL (2000). Sharable Object Reference Model, SCORM. Disponible en: 
Burgos, D. y Koper, R. (2005). Comunidades virtuales, grupos y proyectos de investigación sobre IMS Learning Design. Status quo, factores clave y retos inmediatos. RELIEVE: v. 11, n. 2, p. 189-200.

http://www.uv.es/RELIEVE/v11n2/RELIEVEv11n2_6.htm

http://www.adlnet.org/index.cfm?fuseaction=scor mabt. Último acceso: 29 de abril de 2005

Burgos, D., Berbegal, N., Griffiths, D., Tattersall, C., Koper, R. (2005) IMS Learning Design: How specifications could change the current e-learning landscape. e-Learning World, issue 2, MarchApril 2005. ISSN: 1811-069X. Moscow, Russia: Moscow State University of Economics, Statis- tics and Informatics - MESI. Disponible en: http://hdl.handle.net/1820/354. Último acceso: 1 de agosto de 2005

Koper, Rob; Tattersall, Colin (2005) Learning Design: A Handbook on Modelling and Delivering Networked Education and Training. Germany: Springer Verlag

\section{NOTAS}

[1] UNFOLD Project, http://www.unfold-project.net/

[2] Learning Networks for Learning Design, http://moodle.learningnetworks.org/

[3] Open University of The Netherlands, http://www.ou.nl/

\section{ABOUT THE AUTHORS / SOBRE LOS AUTORES}

Daniel Burgos (Daniel.Burgos@ou.nl) (Spain, 1970) joined the OUNL in 2004 as a Researcher in Educational Technology, after working fourteen years as a teacher, multimedia developer and academic manager in Europe and South America, also with his own company. He is currently focused on IMS Learning Design, adaptive learning, contextual feedback and e-learning systems and he is involved in UNFOLD Project, managing the website moodle.learningnetworks.org. He has written thirteen books and more than one hundred of articles for general magazines about multimedia and Internet issues and holds degrees in Computer Science, Communication, Education and Business Administration.

Rob Koper (rob.koper@ou.nl) Prof. dr E.J.R. Koper (The Netherlands, 1957) is Professor of Educational Technology at the Educational Expertise Centre (OTEC) of the Open University of the Netherlands from 1998, where he leads the RTD programme into learning technologies at OTEC, a team of around 30 researchers \& technology developers. In the eighties and early nineteenths he was leading different innovation projects related to the use of ICT in education in the Netherlands; head of learning technology infrastructure and application development (e-learning infrastructures, educational portals, educational software development, etc.

\section{ARTICLE RECORD / FICHA DEL ARTÍCULO}

\begin{tabular}{|l|l|}
\hline $\begin{array}{l}\text { Reference / } \\
\text { Referencia }\end{array}$ & $\begin{array}{l}\text { Burgos, Daniel y Koper, Rob (2005). Comunidades virtuales, grupos y proyectos de investigación sobre ims learning design. Status } \\
\text { quo, factores clave y retos inmediatos. Revista ELectrónica de Investigación y EValuación Educativa, v. 11, n. 2. } \\
\text { http://www.uv.es/RELIEVE/v11n2/RELIEVEv11n2_6.htmConsultado en (poner fecha). }\end{array}$ \\
\hline $\begin{array}{l}\text { Title / Títu- } \\
\text { lo }\end{array}$ & $\begin{array}{l}\text { Comunidades virtuales, grupos y proyectos de investigación sobre ims learning design. Status quo, factores clave } \\
\text { y retos inmediatos [Virtual communities, research groups and projects on IMS Learning Design. State of the art, } \\
\text { key factors and forthcoming challenges] }\end{array}$ \\
\hline $\begin{array}{l}\text { Authors / } \\
\text { Autores }\end{array}$ & Daniel Burgos \& Rob Koper \\
\hline Review / & Revista ELectrónica de Investigación y EValuación Educativa (RELIEVE), v. 11, n. 2 \\
\hline
\end{tabular}


Burgos, D. y Koper, R. (2005). Comunidades virtuales, grupos y proyectos de investigación sobre IMS Learning Design. Status quo, factores clave y retos inmediatos. RELIEVE: v. 11, n. 2, p. 189-200.

http://www.uv.es/RELIEVE/v11n2/RELIEVEv11n2_6.htm

\begin{tabular}{|c|c|}
\hline Revista & \\
\hline ISSN & $1134-4032$ \\
\hline $\begin{array}{l}\text { Publication } \\
\text { date / } \\
\text { Fecha de } \\
\text { publicación }\end{array}$ & $\begin{array}{l}2005 \text { (Reception Date: } 2005 \text { August 12; Approval Date: } 2005 \text { November 30; Publication Date: } 2005 \text { De- } \\
\text { cember } 1 \text { ) }\end{array}$ \\
\hline $\begin{array}{l}\text { Abstract / } \\
\text { Resumen }\end{array}$ & $\begin{array}{l}\text { We carry out a report showing the state of the art about virtual communities, research groups and projects fo- } \\
\text { cused on the e-learning specification IMS Learning Design or directly related to it. This specification is currently } \\
\text { becoming the most flexible and supported de facto standard for modelling full learning processes, as a comple- } \\
\text { ment for any structure of educational contents. Afterwards, as a consequence of the previous study, we develop a } \\
\text { reading and a further analysis of the current panorama, and describe the key factors that show the relevance and } \\
\text { impact of IMS Learning Design and also the main forthcoming challenges. } \\
\text { Realizamos una descripción del estado del arte sobre las comunidades virtuales, los grupos de trabajo y los } \\
\text { proyectos de investigación centrados en la especificación de e-learning IMS Learning Design o desarrollados en } \\
\text { torno a ella. Esta especificación se está convirtiendo de facto en el estándar más versátil y respaldado para mode- } \\
\text { lar procesos completos de aprendizaje como complemento de estructuras de contenidos educativos. Posterior- } \\
\text { mente, y como consecuencia del estudio, desarrollamos un análisis y lectura del panorama actual con una indica- } \\
\text { ción de los factores clave que muestran su impacto y relevancia y los principales retos a abordar en un futuro } \\
\text { inmediato. }\end{array}$ \\
\hline $\begin{array}{l}\text { Keywords } \\
\text { Descriptores }\end{array}$ & $\begin{array}{l}\text { IMS Learning Design, e-learning standard, interoperability, re-use, unit of learning } \\
\text { IMS Learning Design, estándar e-learning, interoperabilidad, reutilización, unidad de aprendizaje }\end{array}$ \\
\hline \begin{tabular}{l|} 
Institution / \\
Institución
\end{tabular} & Open University of The Netherlands \\
\hline $\begin{array}{l}\text { Publication } \\
\text { site / } \\
\text { Dirección }\end{array}$ & http://www.uv.es/RELIEVE \\
\hline $\begin{array}{l}\text { Language / } \\
\text { Idioma }\end{array}$ & Español (Title, abstract and keywords in english ) \\
\hline
\end{tabular}

\section{Revista ELectrónica de Investigación y EValuación Educativa (RELIEVE)}

\section{[ ISSN: 1134-4032 ]}

(C) Copyright, RELIEVE. Reproduction and distribution of this articles it is authorized if the content is no modified and their origin is indicated (RELIEVE Journal, volume, number and electronic address of the document).

(C) Copyright, RELIEVE. Se autoriza la reproducción y distribución de este artículo siempre que no se modifique el contenido y se indique su origen (RELIEVE, volumen, número y dirección electrónica del documento). 